\title{
The Domestic Shape of Al: A Reflection on Virtual Assistants
}

\section{Abstract}

Artificial intelligence is more-or-less covertly entering our lives and houses. In this context, virtual assistants such as the renowned Amazon Alexa and the products that embed them, are the most representative of the first wave of materialization of artificial intelligence in the domestic domain. We focus on these products, studying their tangible form and appearance as well as the interaction modalities of 10 domestic virtual assistants already in the market or close to be. Our analysis highlights two main approaches in terms of shape: on the one hand $\mathrm{Al}$ is embedded into common home appliances - usually speakers - while, on the other hand, robot-like assistants are taking ground. Concurrently, we portray a still immature reflection on this issue from a design perspective. We highlight a frequently poor translation of functions into tangible shapes as well as a lack of reflection on interaction design basics, such as: input and output modalities, feedback systems and functions discoverability. The subsequent discussion frames the results in a broader reflection about shape, function and meaning paving the way to alternative paths to follow for the design of future home virtual assistants.

\section{Keywords}

Al, Interaction Design, Home Virtual Assistants, Tangible Forms, Interaction Modalities

\section{Introduction}

Aware that the world is gradually moving towards the Ubiquitous Computing that Weiser envisioned in 1991 [1], and computation is spreading throughout the physical space and across multiple devices to build environments that help people in their ordinary activities [2], we want to frame the transitional period we are experiencing, looking for a path to follow in design research and practice. In particular we look at those products we consider representative of the first wave of materialization of $\mathrm{Al}$ in the domestic landscape, namely virtual assistants. Today, conversational Al-based agents such as Amazon Alexa, are spread in 100 million houses according to Amazon's SVP [3].

If studies have been carried out on the UX of conversational agents [4] and on how Machine Learning $(M L)$ is/can be integrated into the UX $[5,6]$, the design discipline still has not investigated how $\mathrm{Al}$ and its skills have been materialized into objects. This aspect is the focus of the paper that analyses those products specifically designed to act as domestic virtual assistants.

The aim is to initiate a discussion in the design domain on the tangible appearance of $\mathrm{Al}$-enabled virtual assistants, focusing on the shape these objects have, on how they embody skills and interact with people. 


\subsection{Al-enabled Virtual Assistants: an Analysis}

For years Al has been covertly pervading systems and devices that we use daily, but recently, the exigence to state the existence of $\mathrm{Al}$ technology resulted in specifically designed products. As a consequence, domestic multi-purpose assistants, materialized the smartness of $\mathrm{Al}$ in the shape of smart speakers that can learn through continuous conversations with their owners. Furthermore, in the last years, affordable domestic robots, has entered the market opening a frontier that is closer to the idea traditionally associated with Al: that of sentient robots able to simulate human behavior.

These applications exemplify a double interpretation of $\mathrm{Al}$, which has been animating the scientific debate for years: on the one hand, McCarthy's position focused on the creation of a super brain capable of simulating human behavior while, on the other hand, Engelbart's position based on the amplification of human potential through Al [7].

Taking this dichotomy into consideration, we analyze here ten devices integrating $\mathrm{Al}$ agents (Figure 1), the only in the market with the characteristics listed in the following. They are (i) multipurpose home assistants with no other distinct goal (i.e. elderly assistance, etc.), (ii) specifically designed as first-party hardware, (iii) already commercialized or coming in the near future and (iv) able to control other smart home appliances. In case of families of products, we only considered the first released in their latest version.

The investigation on domestic virtual assistants starts from the analysis of their aesthetics of interaction [8], as evidence of Al embodiment. It encompasses physical appearance, use and interactivity, in preliminary considerations. The study here presented does not aim at evaluating the user experience of the analyzed products, but to portray the state of the art of domestic assistants, aiming at stimulating further reflections.

Hence, neither UX assessment protocols [9] nor usability tests [10] have been employed to analyze the declared features. Products have been evaluated through official videos and documentation from their producers. A method considered suitable, given the aim of the research and that some of the products are still not in the market.

Table 1 lists the products analyzed (arranged by release date of the first model) and crosses the basic parameters we considered for the analysis: (i) physical appearance, (ii) input and output modalities, (iii) feedback systems and (iv) discoverability [11] of functions, specifically considering how proactive those artifacts are.
Fig. 1. Pictures of the analyzed domestic assistants (authors' graphic elaboration of official producers' images)

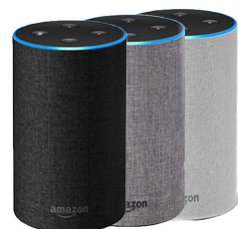

Amazon Echo

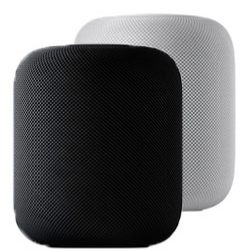

Apple HomePod

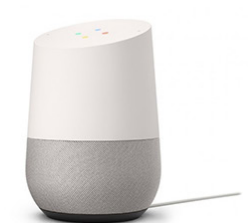

Google Home

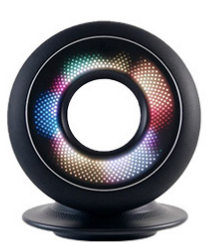

Emotech Olly

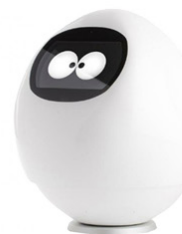

Mji Tapia

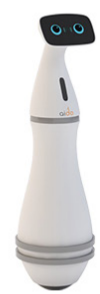

InGen Dynamics Aido

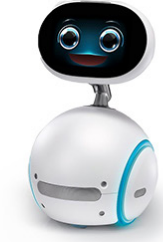

Asus Zenbo

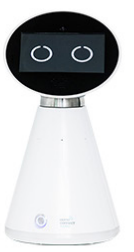

Home Connect

Mykie

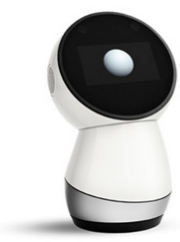

Jibo

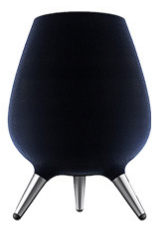

Samsung Galaxy Home 
These parameters have been chosen to analyze and describe, in a very basic manner, the selected products from a product/interaction design standpoint.

A choice motivated by authors' main hypothesis about domestic virtual assistants. Acknowledging the great market success of some of them, this paper aims at showing how immature they are in some respects and at encouraging designers to take on a leading role in identifying a language and a meaning, beside a form.

\section{On the Embodiment of Virtual}

\section{Assistants: A Review}

Physical Appearance. Domestic virtual assistants share quite similar appearance in terms of main colors and materials. They cover the grayscale with white being the most used color, a choice that may be inspired by sci-fi imaginary. On the other hand, black and grey derive from traditional hi-fi aesthetics, underlying their main functionality as speakers. Plastic is the predominant material, while fabrics are getting ground, seeking a better integration within the domestic environment.

Shape is the most significant feature. The review highlights two main formal paths: on the one hand, there are smart objects following simple and mainly regular shapes; on the other, assembled bodies are built according to the geometric addition of solids [12], in four cases looking for a characterization as human/ animal like shape with a recognizable head and body. The separation of formal outcomes also highlights a different functional purpose, giving proof of the fact that the intended tasks have a relationship with the final shape: smart speakers are the first embodiment of Al, and as such a natural outcome for a speaking technology. Home Pod, for example, is totally focused on audio

Table 1. Comparative analysis of home virtual assistants

\begin{tabular}{|c|c|c|c|c|c|c|c|c|c|c|c|}
\hline \multicolumn{2}{|c|}{ DEVICES } & $\begin{array}{c}\text { Amazon } \\
\text { Echo }\end{array}$ & $\begin{array}{l}\text { Google } \\
\text { Home }\end{array}$ & Mji Tapia & $\begin{array}{l}\text { Asus } \\
\text { Zenbo }\end{array}$ & Jibo & $\begin{array}{c}\text { Apple } \\
\text { Home Pod }\end{array}$ & $\begin{array}{l}\text { Emotech } \\
\text { Olly }\end{array}$ & $\begin{array}{c}\text { InGen } \\
\text { Dynamics } \\
\text { Aido }\end{array}$ & $\begin{array}{c}\text { Home } \\
\text { Connect } \\
\text { Mykie }\end{array}$ & $\begin{array}{c}\text { Samsung } \\
\text { Galaxy } \\
\text { Home }\end{array}$ \\
\hline \multicolumn{2}{|c|}{ Year of production } & 2014 & 2016 & 2016 & 2017 & 2017 & 2018 & 2018 & 2019 & tba & tba \\
\hline \multirow{3}{*}{$\begin{array}{l}\text { PHYSICAL } \\
\text { APPEARANCE }\end{array}$} & Simple Shape & ○ & ○ & $\bullet$ & & & ○ & & & & \\
\hline & Assembled solids & & & & $\bullet$ & - & & $\bullet$ & $\bullet$ & - & $\bullet$ \\
\hline & Main Color \& Material & $B / G, F$ & $W+G, P$ & W, P & $W, P$ & $W+G, P$ & W/B, P & $B, P$ & W, P & $W, P$ & $B, P$ \\
\hline \multirow{2}{*}{ DISCOVERABILITY } & Proactive & & & ○ & & & & - & & 0 & \\
\hline & Non Proactive & - & $\bullet$ & & - & - & - & & - & & - \\
\hline \multirow{7}{*}{ INPUT } & Voice & - & $\bullet$ & $\bullet$ & - & 0 & - & - & - & 0 & - \\
\hline & App & - & $\bullet$ & - & - & $\bullet$ & ○ & - & ○ & $\bullet$ & - \\
\hline & Buttons & - & - & - & ? & - & & ? & - & - & \\
\hline & Touch Surface & & O & & & & O & & & & 0 \\
\hline & Touch Display & & & - & - & - & & & - & - & \\
\hline & Vision (camera) & & & - & - & & & ○ & - & 0 & \\
\hline & Touch & & & & & 0 & & & 0 & & \\
\hline \multirow{4}{*}{ OUTPUT } & Other Device Action & - & - & - & - & - & - & - & - & - & - \\
\hline & Audio & - & - & - & $\bullet$ & - & - & $\bullet$ & - & - & $\bullet$ \\
\hline & Video & & & $\bullet$ & ○ & - & & & - & $\bullet$ & \\
\hline & Movement & & & - & - & - & & - & - & 0 & \\
\hline \multirow{4}{*}{ FEEDBACK } & Lights & - & - & & - & 0 & 0 & - & & & - \\
\hline & Voice & 0 & ○ & ○ & ○ & 0 & 0 & ○ & ○ & 0 & 0 \\
\hline & Movement & & & - & & $\bullet$ & & - & & & \\
\hline & Display & & & ○ & 0 & ○ & & & 0 & ○ & \\
\hline
\end{tabular}


quality and it looks like nothing but a speaker. On the contrary, devices aiming at establishing a social contact assume a more anthropomorphic shape. For instance, Aido is thought of as a butler and its height and shape nurture this concept. So, functions highly influence the general, formal configuration of the object, also in terms of dimensions. Speaker-shaped assistants are nothing more than discreet ornaments, while those with social qualities increase to the dimensions of small home appliances or become bigger, in case of a closer human simulation.

The possibility of movement may contribute as well to the embodiment of function: Olly has neither a simple shape nor an anthropomorphic one, but its movements nurture a sense of social connection.

Behavior and Discoverability. Al assistants are based on ML, then evolving according to their owners' preferences is a common feature. Again, what influences the behavior of domestic virtual assistants is their main purpose, once more underlining the distinction between smart speakers and domestic robots.

Most of the assistants, namely smart speakers (Amazon Echo, Google Home, Apple HomePod, Samsung Galaxy Home) and, surprisingly, some robot-like assistants (Zenbo, Jibo and Aido) have a non-proactive behavior: they are unobtrusive and respond only when prompted. On the contrary, three assistants are also proactive and suggest information, activities or contents to their users according to their habits, mood or expected necessities. To better perform their proactivity, they integrate a camera (or more), so that they can relate on more data to foster their suggestions: they not only evaluate noises or routines, but they also read body language and can understand what their users are doing. Furthermore, they can recognize and be triggered just when their users are passing by. The highest point in terms of empathic interaction and proactivity is represented by Olly, which develops and manifests its own personality according to its interlocutor's one. The discoverability of non-proactive objects is really low, and most of their functions remain obscure to the user. This is the case of Alexa: it has thousands of skills, mostly created by third-party developers, but rarely known and used [13]. Interaction. The investigation on interactivity has been limited to input, output, and feedback modalities, referring to Saffer's Systems Design [11]. The main inputs and outputs are vocal, highlighting one of the most important achievements of Al towards a more human interaction. Indeed, Natural Language Processing (NLP) has improved to the point that it can easily understand human requests and answer accordingly. This is the main premise for the rising of digital assistants, which represent the best evidence of this technological accomplishment.

Another feature that all of the devices still share as an input is a through-app interaction: its functionality ranges from basic setup to complete functions (especially for all the smart speakers). Moreover, they also have buttons to perform specific activities: a mutemicrophone button and, respectively, volume up/down buttons or touch surface. On the contrary, robot-like assistants only need the starting one as they have a touch display as a face. Additionally, proactive devices take advantage of their cameras to read body language and gestures as inputs. While, finally, some of them (namely Jibo and Aido) emotionally respond to touch inputs as a result of being cuddled.

In terms of outputs, all the assistants allow the interaction with other home appliances, and the providing of audio contents: web researches, music, podcasts, etc. For the devices having a display or a projector (Aido and Mykie), video contents are also an output, which can be a reproduction of internet sources or an enriching characterization of what they are saying or doing.

Movement is another possible output: it can be the result of a request - dancing (Jibo) or moving across the rooms (Aido and Zenbo) - or just a reinforcement for communication - moving up and down while counting push-ups (Olly).

What is more relevant in an interpersonal-simulated interaction, though, is the feedback system. Relying on [14]'s framework to analyze human-product interaction, it results that $\mathrm{Al}$-enabled assistants offer almost no inherent feedback, as physical actions are required in a very limited manner; then only functional feedbacks (corresponding to the described outputs) and augmented feedbacks characterize the current domestic assistants. In particular, lights, verbal utterances, 
movements and displays reveal the internal state of the object while the function is processing. Almost all of the objects use lighting systems to show their current status, especially the speaker-based assistants, whose choreographies remind those they already have on other devices (for example, it is the case of Google Home bouncing dots). It is to be noticed that - except for Galaxy Home - all the brands have opted for colorful lights, that become particularly expressive in the Olly's custom-built circular LED display: it really underlines the effort of creating a patent communication system through lights.

Another typical feedback of digital assistants comes from their voice: whether in a rigorous or more confidential way, with a robotic or person-like tone, these devices let their users know if and what they have understood before they provide the requested content. This kind of feedback is especially positive in the interaction with anthropomorphic assistants as it gives the impression of being engaged in an actual conversation with a companion, and not just being talking to a machine.

For the devices having a display and being able to move, those are great occasions for feedback: natural and fluid movements can follow the activity or stress the awareness of the robot in its being addressed to its user - for instance turning its head towards the one who is speaking. Displays, instead, are used to show the bot's more or less abstracted eyes, which animate in relation to the user's inputs. Those feedbacks are the most effective in providing a natural interaction, as the machine appears more alive, expressing its own identity. An aspect that is strongly related to feedback, and highly influences the interaction, is feedforward [14]. In the example of virtual assistants, the user (and potentially the designer and/or programmer) does not know what will happen after making a request. In many cases (s)he can only imagine or expect a certain outcome, while one can be absolutely sure of the more usual interactions (through app or buttons) and of the basic and routine commands that (s)he has performed various times. Otherwise, the output of the interaction is unpredictable and - additionally - not immediate: two aspects that may hinder the perceived quality of the interaction.

\section{Reflections on the Embodiment of Al- Enabled Assistants}

The discussion of the formal outcomes cannot be separated from the main functions of the object. As remarked, the shape derives from use and from the relationship that the devices have with their context. Except from its inclusion in the name of some products, the concept of home has been poorly taken in consideration while designing the appearance of those objects. All of them are devices that can be freely set in any environment, and still have not created a connection with our domestic reality: a condition which may contribute to make people perceive them as aliens in their houses. Perhaps, a closer relationship could help users to understand the assistant's benefits more easily. In the meantime, they are interpreted according to their similarity to other, known, objects, or to the abstract expectations that the speculations of our culture have encouraged. Then, it does not come by chance that the most common use of speaker-shaped intelligent assistants is to play music [4], despite the introduction of NLP features opened novel opportunities. On the other hand, devices with a humanoid figure are commonly defined as companions or home managers.

Then, coming to their functionalities, it emerges that users prefer few known commands that actually transform the intelligent assistants into mere executors of routines, especially talking about smart speakers. Hence, the multiple skills they have are rarely used, as discussed by [15] and confirmed by [4] through quantitative and qualitative analysis of use of Alexa. The study by [13] underlines that discoverability in smart speakers is a big issue for designers and it proposes possible solutions to overcome the problem, including context awareness and proactivity. This condition of poor discoverability and weak affordance $[16,17]$ may be due to a deficiency in the embodiment of the skills into tangible products, which often mark a rejection of sensuous curiosity and pleasure [18-20]. In fact, when executing actions, users are prevented from experiencing their inherent effect. In particular, [14]'s framework may be employed to pursue a more intuitive interaction. In order to strengthen its quality, the authors suggest restoring natural couplings between actions and reactions according to six different factors. At the moment, the most promising and easy-to- 
integrate solutions are related to the expression of interaction: the output modality could be a reflection of the conditions in which the request is performed, such as the user's mood or the moment of the day [21]. Currently just three of the analyzed devices (Olly, Mykie and Tapia) feature these functionalities, but it could give true value to the overall interaction and utility of the assistants and, possibly, inform their shape.

One hypothesis is that a humanized aspect and behavior can make an $\mathrm{Al}$-enabled device truly appear like a domestic assistant. Probably its association with a human being sets the conditions for a more natural interaction and makes it simple to imagine that the object has covered skills and it is likely to have a proactive role in our daily life. Yet, we are incline to anthropomorphize everything [21], and functions - made explicit through proactivity -, shape and movements are what actually seems to facilitate users in terms of discoverability and, therefore, interaction. Furthermore, the behavior of those devices characterizes them with well-defined identities.

In the light of this reasoning, another consideration can be pointed out: a distinctive trait of some anthropomorphic assistants is what defines them as mutuality of influences systems [18], meaning that they are sensitive to perceptual crossing. It is not only the user who has to perceive the object of interaction, but it also has to sense the individual who is going to trigger it, preparing and giving notice of this awareness. In this way the interaction becomes expressive, embodied and responsive, even without the use of an interface.

Deeper connections between shape, abilities and behaviors should be developed according to a more mutual relationship between objects and people, trying to understand what it is really about.

Facing the complexity of these devices and their role in the ordinary life, another issue designers must reflect on is the materialization of a great amount of skills, especially if the only means of interaction is conversation. In fact, most of the analyzed assistants feature a companion app to be accessed through smartphone, allowing the personalization of the product as well as a traditional browsing of the skills.
Yet it contradicts one of the premises for a natural interaction: getting rid of interfaces to make the mechanism of the artifact directly available to the system users [22].

Undertaking the same direction, a similar, and perhaps less intuitive, interaction is allowed by the devices integrating a tablet as head, which can be actively used for input and output. An arguable solution that entails an evidently poor investigation about the meaning of interaction.

Analogously, the use of voice as main tangible manifestation of the intelligence behind domestic assistants does not automatically make them real conversational agents, nor it makes it easy to exploit their potential. This condition is made evident by the choice of every producer to integrate added input systems ranging from simple buttons to cameras and complex nested menus to be browsed through bespoke apps. In other words, the conversational agents are frequently thought of as the tip of an iceberg that should make interaction more human and friendlier but, actually, they are still in need of a better definition.

\section{Future Design Scenarios for Virtual Assistants}

The first wave of materialization of $\mathrm{Al}$ into the domestic domain, as described in the introduction, is still profoundly immature in terms of function, language and meaning [23].

Designers are called to manage the coupling of form and ever-increasing functionalities, finding a compromise between expressing them or keeping them implicit. The current situation still mirrors the dichotomy animating the discourse within the field of Al. On the one hand, products like smart speakers materialize $\mathrm{Al}$ into aesthetically appealing objects inhabiting our houses. On the other, objects with varied shapes aim at being perceived as valuable humanoids to help in ordinary life. A dichotomy that permeates all the aspects - with regard to physical appearance, behavior and interaction - analyzed in the study and betrays a still immature reflection from a design perspective, and the need to find an original language. 
Even though the argumentation only considers few initial parameters and presents limitations better addressed in the following, some preliminary considerations may arise. The impression is that the field is still experiencing that period of drunkenness that characterizes the introduction of every new technology [24]. Domestic assistants seem to be in the toy phase having to find their actual contact with reality [25], and design should take a leading role in guiding a humancentered transition towards meaningful products. Present perspectives lead to the integration of $\mathrm{Al}$ discourse into the Interaction Design field. In particular, they share the goal of fostering a more natural interaction, going beyond display-mediated interfaces.

Then, translating $\mathrm{Al}$ in a tangible and domestic form, according to a human-centered and holistic perspective, shape cannot be separated from function and meaning. From what seen, Al-enabled objects could simplify their functional structure so that, from a tangible point of view, the interaction could be more immediate and significant in terms of experience and utility. In this sense, we could be facing three main scenarios: (i) virtual assistants could evolve as self-standing objects, maybe with more specific and limited functions that better translate into a clearly recognizable form; (ii) they could become more accurate humanized robots, taking the role of actual people at service of others; or maybe, (iii) they could be completely dematerialized and spread across other existing appliances and devices throughout the physical environment, with a sensibility for their location and proximity to eachother, accomplishing the ideal of Ubiquitous Computing [22]. The latter, in turn, may open further enquiries about the embodiment, regarding its scale (product or environment) and the way the augmented functionality will be expressed.

Clearly, the study presents several limitations. It takes into account a small sample of products, even if they represent the totality of those responding to the selection criteria. A broader study may include the entire range of products of the producers here analyzed and, eventually, third-party hardware integrating Al agents. Furthermore this initial argumentation, starts from the fundamental traits of a product, but may expand triggering a discussion about current physical materialization of $\mathrm{Al}$ that may comprehend other design matters like UX, or interdisciplinary reflections like social/psychological implications, as the emotional response they may nurture through interaction, as well as experimental studies.

\section{References}

1. Weiser M (1991) The Computer for the 21st Century. Scientific American, 3(265), 94-104.

2. Kaptelinin $\vee$, Nardi BA (2009) Acting with Technology. Activity Theory and Interaction Design. MIT Press, Cambridge, London

3. Bohn D (2019) Exclusive: Amazon says 100 million Alexa devices have been sold. The Verge, https://www.theverge. com/2019/1/4/18168565/amazon-alexa-devices-how-manysold-number-100-million-dave-limp

4. Sciuto A, Saini A, Forlizzi J, Hong Jl (2018) "Hey Alexa, What's Up?": A Mixed-Methods Studies of In-Home Conversational Agent Usage. In: Proceedings of the 2018 Designing Interactive Systems Conference (pp. 857-868). ACM.

5. Dove G, Halskov K, Forlizzi J, Zimmerman J (2017) UX Design Innovation: Challenges for Working with Machine Learning as a Design Material. In Proceedings of the 2017 $\mathrm{CHI}$ Conference on Human Factors in Computing Systems (pp. 278-288). ACM.

6. Yang Q, Sciuto A, Zimmerman J, et al (2018) Investigating How Experienced UX Designers Effectively Work with Machine Learning. In Proceedings of the 2018 Designing Interactive Systems Conference (pp. 585-596). ACM.

7. Winograd T (2006) Shifting viewpoints: Artificial intelligence and human-computer interaction. Artificial Intelligence, 170(18), 1256-1258

8. Graves Petersen M, Hallnäs L, Jacob RJK (2008) Introduction to Special Issue on the Aesthetics of Interaction. ACM Transactions on Computer-Human Interaction, 15(4).

9. Hassenzahl M (2001) The Effect of Perceived Hedonic Quality on Product Appealingness. International Journal of Human-Computer Interaction 13(4), 481-499.

10. Brooke J (2013) SUS: A Retrospective. Journal of Usability Studies 8(2), 29-40

11. Saffer D (2009) Designing for Interaction: Creating Innovative Applications and Devices, 2nd edition. New Riders Pub.

12. Van Onck A (1994) Design. Il senso delle forme dei prodotti. Lupetti Editori di Comunicazione.

13. White RW (2018) Skill Discovery in Virtual Assistants. 
Communications. ACM, 61(11), 106-113.

14. Wensveen S, Djajadiningrat JP, Overbeeke CJ (2004) Interaction frogger: A design framework to couple action and function through feedback and feedforward. In: Processes, Practices, Methods, and Techniques.

15. Kinsella B (2018) Amazon Alexa Now Has 50,000 Skills Worldwide, works with 20,000 Devices, Used by 3,500 Brands. Accessed 26 Feb 2019: https://voicebot. ai/2018/09/02/amazon-alexa-now-has-50000-skillsworldwide-is-on-20000-devices-used-by-3500-brands/.

16. Gibson JJ (1979) The Ecological Approach to Visual Perception, 1 edition. Taylor \& Francis.

17. Norman D (1988) The Psychology of Everyday Things. Basic Books.

18. Marti P (2010) Perceiving While Being Perceived. International Journal of Design, 4(2).

19. Norman D (2004) Emotional design: Why we love (or hate) everyday things. Basic Books.

20. Jordan P (2000) Designing Pleasurable Products: An Introduction to the New Human Factors. Taylor and Francis.

21. Pavliscak P (2017) Emotionally Intelligent Design. Design Is [Emotion]

22. Dourish P (2001) Where the Action Is. The Foundations of Embodied Interaction. MIT Press.

23. Kolko J (2011) Thoughts on Interaction Design, Second Edition. Morgan Kaufmann

24. Antonelli, P. (2018, February 8). Al Is Design's Latest Material. Retrieved from https://design.google/library/aidesigns-latest-material/

25. Levinson P (1977) Toy, Mirror, and Art: The Metamorphosis of Technological Culture. Learning Cyberspace 34,151-167 